\title{
Oscillation Criteria for Differential Equations with Several Retarded Arguments
}

\author{
By \\ G. Infante, R. Koplatadze and I. P. Stavroulakis \\ (Università della Calabria, Italy, Tbilisi State University, Georgia and \\ University of Ioannina, Greece)
}

\begin{abstract}
Consider the first-order linear differential equation with several retarded arguments $x^{\prime}(t)+\sum_{i=1}^{m} p_{i}(t) x\left(\tau_{i}(t)\right)=0, t \geq t_{0}$, where the functions $p_{i}, \tau_{i} \in$ $C\left(\left[t_{0}, \infty\right), \boldsymbol{R}^{+}\right)$, for every $i=1,2, \ldots, m, \tau_{i}(t) \leq t$ for $t \geq t_{0}$ and $\lim _{t \rightarrow \infty} \tau_{i}(t)=\infty$. In this paper the state-of-the-art on the oscillation of all solutions to these equations is reviewed and new sufficient conditions for the oscillation are established, especially in the case of nonmonotone arguments. Examples illustrating the results are given.

Key Words and Phrases. Oscillation, Retarded, Differential equations, Nonmonotone arguments.

2010 Mathematics Subject Classification Numbers. Primary 34K11; Secondary $34 \mathrm{~K} 06$.
\end{abstract}

\section{Introduction}

Consider the differential equation with several non-monotone retarded arguments

$$
x^{\prime}(t)+\sum_{i=1}^{m} p_{i}(t) x\left(\tau_{i}(t)\right)=0, \quad t \geq t_{0},
$$

where the functions $p_{i}, \tau_{i} \in C\left(\left[t_{0}, \infty\right), \boldsymbol{R}^{+}\right)$, for every $i=1,2, \ldots, m$, (here $\left.\boldsymbol{R}^{+}=[0, \infty)\right), \tau_{i}(t) \leq t$ for $t \geq t_{0}$ and $\lim _{t \rightarrow \infty} \tau_{i}(t)=\infty$.

Let $\quad T_{0} \in\left[t_{0},+\infty\right), \quad \tau(t)=\min \left\{\tau_{i}(t): i=1, \ldots, m\right\} \quad$ and $\quad \tau_{(-1)}(t)=$ $\sup \{s: \tau(s) \leq t\}$. By a solution of the equation (1.1) we understand a function $x \in C\left(\left[T_{0},+\infty\right) ; R\right)$, continuously differentiable on $\left[\tau_{(-1)}\left(T_{0}\right),+\infty\right)$ and that satisfies $(1.1)$ for $t \geq \tau_{(-1)}\left(T_{0}\right)$. Such a solution is called oscillatory if it has arbitrarily large zeros, and otherwise it is called nonoscillatory.

In the special case where $m=1$ equation (1.1) reduces to the equation

$$
x^{\prime}(t)+p(t) x(\tau(t))=0, \quad t \geq t_{0},
$$

where the functions $p, \tau \in C\left(\left[t_{0}, \infty\right), \boldsymbol{R}^{+}\right), \tau(t) \leq t$ for $t \geq t_{0}$ and $\lim _{t \rightarrow \infty} \tau(t)$ $=\infty$. 
For the general theory of these equations the reader is referred to $[6,9,11$, 12, 23].

The problem of establishing sufficient conditions for the oscillation of all solutions to the differential equation (1.2) has been the subject of many investigations. See, for example, $[1,2,3,4,5,6,7,14,15,16,17,18,19,20$, $21,24,25,26,27,28,29]$ and the references cited therein.

In this paper we present the state-of-the-art on the oscillation of all solutions to the equation (1.1) in the case of several non-monotone arguments and especially when the well known oscillation conditions (for $m=1$ )

$$
\limsup _{t \rightarrow \infty} \int_{\tau(t)}^{t} p(s) d s>1 \quad \text { and } \quad \liminf _{t \rightarrow \infty} \int_{\tau(t)}^{t} p(s) d s>\frac{1}{e} .
$$

are not satisfied.

\section{Oscillation criteria for equation (1.1)}

The first systematic study for the oscillation of all solutions to the equation (1.2) was made by Myshkis. In 1950 [25] he proved that every solution oscillates if

$$
\limsup _{t \rightarrow \infty}[t-\tau(t)]<\infty \quad \text { and } \quad \liminf _{t \rightarrow \infty}[t-\tau(t)] \liminf _{t \rightarrow \infty} p(t)>\frac{1}{e} .
$$

In 1972, Ladas, Lakshmikantham and Papadakis [21] proved that the same conclusion holds if in addition $\tau$ is a non-decreasing function and

$$
A:=\limsup _{t \rightarrow \infty} \int_{\tau(t)}^{t} p(s) d s>1 .
$$

In 1979, Ladas [20] established integral conditions for the oscillation of the equation (1.2) with constant delay, while in 1982, Koplatadze and Canturija [17] established the following result. If

$$
\mathfrak{a}:=\liminf _{t \rightarrow \infty} \int_{\tau(t)}^{t} p(s) d s>\frac{1}{e},
$$

then all solutions of the equation (1.2) oscillate; if

$$
\limsup _{t \rightarrow \infty} \int_{\tau(t)}^{t} p(s) d s<\frac{1}{e},
$$

then the equation (1.2) has a non-oscillatory solution.

Concerning the constants 1 and $1 / e$ which appear in the conditions (2.1), (2.2) and (2.3), in 2011, Berezansky and Braverman [2] established the following: 
Theorem 2.1 ([2, Theorem 2.5]). For any $\alpha \in(1 / e, 1)$ there exists a nonoscillatory equation

$$
x^{\prime}(t)+p(t) x(t-\tau)=0, \quad \tau>0
$$

with $p(t) \geq 0$ such that

$$
\limsup _{t \rightarrow \infty} \int_{t-\tau}^{t} p(s) d s=\alpha .
$$

Also, in 2011, Braverman and Karpuz [3] investigated equation (1.2) in the case of a general argument ( $\tau$ is not assumed non-decreasing as in (2.1)) and proved that:

Theorem 2.2 ([3, Theorem 1]). There is no constant $K>0$ such that

$$
\limsup _{t \rightarrow \infty} \int_{\tau(t)}^{t} p(s) d s>K
$$

implies oscillation of equation (1.2) for arbitrary (not necessarily non-decreasing) argument $\tau(t) \leq t$.

Remark 2.1. Observe that, because of the condition (2.3), the constant $K$ in (2.4) makes sense for $K>1 / e$.

Moreover in [3] the above condition (2.1) was improved as follows.

Theorem 2.3 ([3, Corollary 1]). Assume that

$$
B:=\limsup _{t \rightarrow \infty} \int_{\sigma(t)}^{t} p(s) \exp \left\{\int_{\tau(s)}^{\sigma(t)} p(\xi) d \xi\right\} d s>1,
$$

where $\sigma(t)=\sup _{s \leq t} \tau(s), \quad t \geq t_{0}$. Then all solutions of the equation (1.2) oscillate.

For equations with several arguments the following results have been established.

In 1982, Ladas and Stavroulakis [22], (see also in 1984, Arino, Gyori and Jawhari [1]), studied the equation with several constant retarded arguments of the form

$$
x^{\prime}(t)+\sum_{i=1}^{m} p_{i}(t) x\left(t-\tau_{i}\right)=0, \quad t \geq t_{0},
$$

under the assumption that $\lim _{\inf } \int_{t \rightarrow \infty} \int_{t-\tau_{i} / 2}^{t} p(s) d s>0, \quad i=1,2, \ldots, m$, and proved that each one of the following conditions 


$$
\liminf _{t \rightarrow \infty} \int_{t-\tau_{i}}^{t} p_{i}(s) d s>\frac{1}{e} \quad \text { for some } i, i=1,2, \ldots, m
$$

$$
\begin{aligned}
\liminf _{t \rightarrow \infty} \int_{t-\tau}^{t} & \sum_{i=1}^{m} p_{i}(s) d s>\frac{1}{e}, \quad \text { where } \tau=\min \left\{\tau_{1}, \tau_{2}, \ldots, \tau_{m}\right\}, \\
& {\left[\prod_{i=1}^{m}\left(\sum_{j=1}^{m} \liminf _{t \rightarrow \infty} \int_{t-\tau_{j}}^{t} p_{i}(s) d s\right)\right]^{1 / m}>\frac{1}{e}, }
\end{aligned}
$$

or

$$
\begin{aligned}
& \frac{1}{m} \sum_{i=1}^{m}\left(\liminf _{t \rightarrow \infty} \int_{t-\tau_{i}}^{t} p_{i}(s) d s\right) \\
& \quad+\frac{2}{m} \sum_{i<j}^{m}\left[\left(\liminf _{t \rightarrow \infty} \int_{t-\tau_{j}}^{t} p_{i}(s) d s\right)\left(\liminf _{t \rightarrow \infty} \int_{t-\tau_{i}}^{t} p_{i}(s) d s\right)\right]^{1 / 2}>\frac{1}{e}
\end{aligned}
$$

implies that all solutions of equation $\left(1.1^{\prime}\right)$ oscillate. Later in 1996, Li [24] proved that the same conclusion holds if

$$
\liminf _{t \rightarrow \infty} \sum_{i=1}^{m} \int_{t-\tau_{i}}^{t} p_{i}(s) d s>\frac{1}{e}
$$

In 1984, Hunt and Yorke [13], considered the equation with variable coefficients of the form:

$$
x^{\prime}(t)+\sum_{i=1}^{m} p_{i}(t) x\left(t-\tau_{i}(t)\right)=0, \quad t \geq t_{0}
$$

under the assumption that there is a uniform upper bound $\tau_{0}$ on the $\tau_{i}$ s and proved that if

$$
\liminf _{t \rightarrow \infty} \sum_{i=1}^{m} \tau_{i}(t) p_{i}(t)>\frac{1}{e}
$$

then all solutions of the equation $\left(1.1^{\prime \prime}\right)$ oscillate.

In 1984, Fukagai and Kusano [8], for the equation (1.1) established the following theorem.

Theorem 2.4 ([8, Theorem 1' (i)]). Consider equation (1.1) and assume that there is a continuous non-decreasing function $\tau^{*}(t)$ such that $\tau_{i}(t) \leq \tau^{*}(t) \leq t$ for 
$t \geq t_{0}, 1 \leq i \leq m . \quad$ If

$$
\liminf _{t \rightarrow \infty} \int_{\tau^{*}(t)}^{t} \sum_{i=1}^{m} p_{i}(s) d s>\frac{1}{e},
$$

Then all solutions of the equation (1.1) oscillate. If, on the other hand, there exists a continuous non-decreasing function $\tau_{*}(t)$ such that $\tau_{*}(t) \leq \tau_{i}(t)$ for $t \geq t_{0}$, $1 \leq i \leq m, \lim _{t \rightarrow \infty} \tau_{*}(t)=\infty$ and

$$
\int_{\tau_{*}(t)}^{t} \sum_{i=1}^{m} p_{i}(s) d s \leq \frac{1}{e}
$$

for all sufficiently large $t$, then the equation (1.1) has a non-oscillatory solution.

In 2000, Grammatikopoulos, Koplatadze and Stavroulakis [10] improved the above results as follows:

Theorem 2.5 ([10, Theorems 2.6]). Assume that the functions $\tau_{i}$ are nondecreasing for all $i \in\{1, \ldots, m\}$,

$$
\int_{0}^{\infty}\left|p_{i}(t)-p_{j}(t)\right| d t<+\infty, \quad i, j=1, \ldots, m
$$

and

$$
\liminf _{t \rightarrow \infty} \int_{\tau_{i}(t)}^{t} p_{i}(s) d s=\beta_{i}>0, \quad i=1, \ldots, m
$$

If

$$
\sum_{i=1}^{m}\left(\liminf _{t \rightarrow \infty} \int_{\tau_{i}(t)}^{t} p_{i}(s) d s\right)>\frac{1}{e}
$$

then all solutions of the equation (1.1) oscillate.

\section{New oscillation results}

Observe that all the above mentioned oscillation conditions (2.6)-(2.12) involve lim inf only and coincide with the condition (2.2) in the case that $m=1$. It is obvious that there is a gap between the conditions (2.1) and (2.2) when the limit $\lim _{t \rightarrow \infty} \int_{\tau(t)}^{t} p(s) d s$ does not exist. Moreover, in view of Theorem 2.2, it is an interesting problem to investigate equation (1.1) with non-monotone arguments and derive sufficient oscillation conditions, involving lim sup (as the 
condition (2.1) for the equation (1.2) with one argument), which is the main objective in the following.

Theorem 3.1. Assume that there exist non-decreasing functions $\sigma_{i} \in$ $C\left(\left[t_{0},+\infty\right)\right)$ such that

$$
\tau_{i}(t) \leq \sigma_{i}(t) \leq t \quad(i=1, \ldots, m),
$$

and

$$
\begin{aligned}
\limsup _{t \rightarrow+\infty} \prod_{j=1}^{m} & {\left[\prod _ { i = 1 } ^ { m } \int _ { \sigma _ { j } ( t ) } ^ { t } p _ { i } ( s ) \operatorname { e x p } \left(\int_{\tau_{i}(s)}^{\sigma_{i}(t)} \sum_{i=1}^{m} p_{i}(\xi)\right.\right.} \\
& \left.\left.\times \exp \left(\int_{\tau_{i}(\xi)}^{\xi} \sum_{i=1}^{m} p_{i}(u) d u\right) d \xi\right) d s\right]^{1 / m}>\frac{1}{m^{m}} .
\end{aligned}
$$

Then all solutions of the equation (1.1) oscillate.

Proof. Assume, for the sake of contradiction, that the retarded equation (1.1) admits a non-oscillatory solution $x(t)$. Since $-x(t)$ is also a solution to (1.1), we can confine ourselves only to the case that $x(t)$ is an eventually positive solution of the equation (1.1). Then there exists $t_{1}>t_{0}$ such that $x(t), x\left(\tau_{i}(t)\right)$, $x\left(\sigma_{i}(t)\right)>0(i=1,2 \ldots, m)$ for all $t \geq t_{1}$. Therefore, from equation (1.1) it follows that $x^{\prime}(t) \leq 0$ for all $t \geq t_{1}$ and consequently $x(t)$ is non-increasing. From (1.1) dividing by $x(t)$ and integrating from $s$ to $t$ for sufficiently large $t$ and $s$, we have

$$
x(s)=x(t) \exp \left\{\int_{s}^{t} \sum_{i=1}^{m} p_{i}(\xi) \frac{x\left(\tau_{i}(\xi)\right)}{x(\xi)} d \xi\right\} \quad \text { for } t \geq s .
$$

Integrating (1.1) from $\sigma_{j}(t)$ to $t$, for sufficiently large $t$, we have

$$
x\left(\sigma_{j}(t)\right) \geq \sum_{i=1}^{m} \int_{\sigma_{j}(t)}^{t} x\left(\tau_{i}(s)\right) p_{i}(s) d s .
$$

On the other hand from (3.3), taking into account (3.1) and the fact that the function $x(t)$ is non-increasing, for sufficiently large $t$, we obtain

$$
\frac{x\left(\tau_{i}(t)\right)}{x(t)}=\exp \left\{\int_{\tau_{i}(t)}^{t} \sum_{i=1}^{m} p_{i}(\xi) \frac{x\left(\tau_{i}(\xi)\right)}{x(\xi)} d \xi\right\} \geq \exp \left\{\int_{\tau_{i}(t)}^{t} \sum_{i=1}^{m} p_{i}(\xi) d \xi\right\}
$$

and

$$
x\left(\tau_{i}(s)\right) \geq x\left(\sigma_{i}(t)\right) \exp \left\{\int_{\tau_{i}(s)}^{\sigma_{i}(t)} \sum_{i=1}^{m} p_{i}(\xi) \exp \left(\int_{\tau_{i}(\xi)}^{\xi} \sum_{i=1}^{m} p_{i}(u) d u\right) d \xi\right\} .
$$


Combining the last three inequalities (3.4), (3.5) and (3.6), and using the arithmetic mean-geometric mean inequality, we get

$$
\begin{aligned}
x\left(\sigma_{j}(t)\right) \geq & \sum_{i=1}^{m} \int_{\sigma_{j}(t)}^{t} x\left(\sigma_{i}(t)\right) \\
& \times \exp \left\{\int_{\tau_{i}(s)}^{\sigma_{i}(t)} \sum_{i=1}^{m} p_{i}(\xi) \exp \left(\int_{\tau_{i}(\xi)}^{\xi} \sum_{i=1}^{m} p_{i}(u) d u\right) d \xi\right\} p_{i}(s) d s \\
= & \sum_{i=1}^{m} x\left(\sigma_{i}(t)\right) \\
& \times \int_{\sigma_{j}(t)}^{t} \exp \left\{\int_{\tau_{i}(s)}^{\sigma_{i}(t)} \sum_{i=1}^{m} p_{i}(\xi) \exp \left(\int_{\tau_{i}(\xi)}^{\xi} \sum_{i=1}^{m} p_{i}(u) d u\right) d \xi\right\} p_{i}(s) d s \\
\geq & m\left[\prod_{i=1}^{m} x\left(\sigma_{i}(t)\right)\right]^{1 / m} \times\left[\prod_{i=1}^{m} \int_{\sigma_{j}(t)}^{t} p_{i}(s)\right. \\
& \left.\times \exp \left\{\int_{\tau_{i}(s)}^{\sigma_{i}(t)} \sum_{i=1}^{m} p_{i}(\xi) \exp \left(\int_{\tau_{i}(\xi)}^{\xi} \sum_{i=1}^{m} p_{i}(u) d u\right) d \xi\right\} d s\right]^{1 / m}, \\
& j=1, \ldots, m,
\end{aligned}
$$

and taking the product on both sides of the last inequality, we find

$$
\begin{aligned}
w(t) \geq m^{m} w(t) \prod_{j=1}^{m} & {\left[\prod _ { i = 1 } ^ { m } \int _ { \sigma _ { j } ( t ) } ^ { t } p _ { i } ( s ) \operatorname { e x p } \left\{\int_{\tau_{i}(s)}^{\sigma_{i}(t)} \sum_{i=1}^{m} p_{i}(\xi)\right.\right.} \\
& \left.\left.\times \exp \left(\int_{\tau_{i}(\xi)}^{\xi} \sum_{i=1}^{m} p_{i}(u) d u\right) d \xi\right\} d s\right]^{1 / m}
\end{aligned}
$$

where $w(t)=\prod_{j=1}^{m} x\left(\sigma_{j}(t)\right)$. That is,

$$
\begin{aligned}
\limsup _{t \rightarrow+\infty} \prod_{j=1}^{m} & {\left[\prod _ { i = 1 } ^ { m } \int _ { \sigma _ { j } ( t ) } ^ { t } p _ { i } ( s ) \operatorname { e x p } \left(\int_{\tau_{i}(s)}^{\sigma_{i}(t)} \sum_{i=1}^{m} p_{i}(\xi)\right.\right.} \\
& \left.\left.\times \exp \left(\int_{\tau_{i}(\xi)}^{\xi} \sum_{i=1}^{m} p_{i}(u) d u\right) d \xi\right) d s\right]^{1 / m} \leq \frac{1}{m^{m}},
\end{aligned}
$$

which contradicts (3.2). The proof of the theorem is complete.

For the next theorem we need the following lemma. 
Lemma 3.1. Let

$$
\liminf _{t \rightarrow+\infty} \int_{\tau_{i}(t)}^{t} p_{i}(s) d s=p_{i}>0 \quad(i=1, \ldots, m) .
$$

and $x(t)$ be an eventually positive solution of the equation (1.1). Then

$$
\liminf _{t \rightarrow+\infty} \frac{x\left(\tau_{i}(t)\right)}{x(t)} \geq \lambda_{i}^{*},
$$

where $\lambda_{i}^{*}$ is the smallest root of the equation

$$
e^{p_{i} \lambda}=\lambda
$$

Proof. Let $\varepsilon \in\left(0, p_{i}\right)$. First we show that

$$
\liminf _{t \rightarrow+\infty} \frac{x\left(\tau_{i}(t)\right)}{x(t)} \geq \lambda_{i}^{*}(\varepsilon),
$$

where $\lambda_{i}^{*}(\varepsilon)$ is the smallest root of the equation

$$
e^{\left(p_{i}-\varepsilon\right) \lambda}=\lambda
$$

By (3.7), for any $\varepsilon>0$, there exist $t_{\varepsilon} \in R_{+}$such that

$$
\int_{\tau_{i}(t)}^{t} p_{i}(s) d s \geq p_{i}-\varepsilon \quad \text { for } t \geq t_{\varepsilon} .
$$

Assume, for the sake of contradiction, that (3.9) is not correct. Then, there exists $\varepsilon_{0}>0$ such that

$$
\frac{e^{\left(p_{i}-\varepsilon\right) \gamma_{i}}}{\gamma_{i}} \geq 1+\varepsilon_{0}
$$

where

$$
\gamma_{i}=\liminf _{t \rightarrow+\infty} \frac{x\left(\tau_{i}(t)\right)}{x(t)}<\lambda_{i}^{*}(\varepsilon)
$$

On the other hand, for any $\delta>0$ there exists $t_{\delta}$ such that

$$
\frac{x\left(\tau_{i}(t)\right)}{x(t)} \geq \gamma_{i}-\delta \quad \text { for } t \geq t_{\delta}
$$

Dividing (1.1) by $x(t)$ and integrating from $\tau_{i}(t)$ to $t$ for sufficiently large $t$, and taking into account (3.10) and the last inequality, we have

$$
\frac{x\left(\tau_{i}(t)\right)}{x(t)} \geq \exp \left(\int_{\tau_{i}(t)}^{t} \frac{x\left(\tau_{i}(s)\right)}{x(s)} p(s) d s\right) \geq e^{\left(p_{i}-\varepsilon\right)\left(\gamma_{i}-\delta\right)} \quad \text { for large } t .
$$


Therefore,

$$
\gamma_{i}=\liminf _{t \rightarrow+\infty} \frac{x\left(\tau_{i}(t)\right)}{x(t)} \geq e^{\left(p_{i}-\varepsilon\right)\left(\gamma_{i}-\delta\right)}
$$

which implies

$$
\gamma_{i} \geq e^{\gamma_{i}\left(p_{i}-\varepsilon\right)}
$$

In view of (3.11), this is a contradiction and therefore (3.9) is true. Since $\lambda_{i}^{*}(\varepsilon) \rightarrow \lambda_{i}^{*}$ as $\varepsilon \rightarrow 0,(3.9)$ implies (3.8) and the proof of the lemma is complete.

Using this lemma, as in Theorem 3.1, we can prove the following.

Theorem 3.2. Let (3.1) be fulfilled and for some non-decreasing functions $\sigma_{i} \in C\left(\left[t_{0},+\infty\right)\right)(i=1, \ldots, m)$

$$
\begin{aligned}
\limsup _{\varepsilon \rightarrow 0+}( & \limsup _{t \rightarrow+\infty} \prod_{j=1}^{m}\left(\prod_{i=1}^{m} \int_{\sigma_{j}(t)}^{t} p_{i}(s)\right. \\
& \left.\left.\times \exp \left(\int_{\tau_{i}(s)}^{\sigma_{i}(t)} \sum_{i=1}^{m}\left(\lambda_{i}^{*}-\varepsilon\right) p_{i}(\xi) d \xi\right) d s\right)^{1 / m}\right)>\frac{1}{m^{m}},
\end{aligned}
$$

where $\lambda_{i}^{*}(i=1, \ldots, m)$ is the smallest root of the equation $\left(3.8^{\prime}\right)$. Then all solutions of the equation (1.1) oscillate.

When $m=1$, that is in the case of the equation (1.2) with one argument, from Theorems 3.1 and 3.2 we have, respectively, the following corollaries.

Corollary 3.1. Let

$$
C:=\limsup _{t \rightarrow+\infty} \int_{\sigma(t)}^{t} p(s) \exp \left(\int_{\tau(s)}^{\sigma(t)} p(\xi) \exp \left(\int_{\tau(\xi)}^{\xi} p(u) d u\right) d \xi\right) d s>1 .
$$

Then all solutions of the equation (1.2) oscillate.

Corollary 3.2. Let

$$
\limsup _{\varepsilon \rightarrow 0}\left(\limsup _{t \rightarrow+\infty} \int_{\sigma(t)}^{t} p(s) \exp \left(\int_{\tau(s)}^{\sigma(t)}\left(\lambda^{*}-\varepsilon\right) p(\xi) d \xi\right) d s\right)>1 .
$$

Then all solutions of the equation (1.2) oscillate.

In the case of monotone arguments we have the following. 
Theorem 3.3. Let $\tau_{i}$ be non-decreasing functions and

$$
\begin{aligned}
\limsup _{t \rightarrow+\infty} \prod_{j=1}^{m}[ & \prod_{i=1}^{m} \int_{\tau_{j}(t)}^{t} p_{i}(s) \exp \left(\int_{\tau_{i}(s)}^{\tau_{i}(t)} \sum_{i=1}^{m} p_{i}(\xi)\right. \\
& \left.\left.\times \exp \left(\int_{\tau_{i}(\xi)}^{\xi} \sum_{i=1}^{m} p_{i}(u) d u\right) d \xi\right) d s\right]^{1 / m}>\frac{1}{m^{m}} .
\end{aligned}
$$

or

$$
\begin{aligned}
& \underset{\varepsilon \rightarrow 0+}{\limsup }\left(\operatorname { l i m s u p } _ { t \rightarrow + \infty } \prod _ { j = 1 } ^ { m } \left(\prod_{i=1}^{m} \int_{\tau_{j}(t)}^{t} p_{i}(s)\right.\right. \\
& \left.\left.\quad \times \exp \left(\int_{\tau_{i}(s)}^{\tau_{i}(t)} \sum_{i=1}^{m}\left(\lambda_{i}^{*}-\varepsilon\right) p_{i}(\xi) d \xi\right) d s\right)^{1 / m}\right)>\frac{1}{m^{m}},
\end{aligned}
$$

where $\lambda_{i}^{*}(i=1, \ldots, m)$ is the smallest root of the equation $\left(3.8^{\prime}\right)$. Then all solutions of the equation (1.1) oscillate.

Corollary 3.3. Let $\tau_{i}$ be non-decreasing functions and

$$
\limsup _{t \rightarrow+\infty} \prod_{j=1}^{m}\left(\prod_{i=1}^{m} \int_{\tau_{j}(t)}^{t} p_{i}(s) d s\right)^{1 / m}>\frac{1}{m^{m}} .
$$

Then all solutions of the equation (1.1) oscillate.

Corollary 3.4. Let $\tau_{i}$ be non-decreasing functions, $p_{i}(t) \geq p(t) \quad(i=$ $1, \ldots, m)$ and

$$
\limsup _{t \rightarrow+\infty} \prod_{j=1}^{m} \int_{\tau_{j}(t)}^{t} p(s) d s>\frac{1}{m^{m}},
$$

Then all solutions of the equation (1.1) oscillate.

Corollary 3.5. Let $\tau_{i}$ be non-decreasing functions, $p_{i}(t) \geq p=$ const and

$$
p^{m} \limsup _{t \rightarrow+\infty} \prod_{i=1}^{m}\left(t-\tau_{i}(t)\right)>\frac{1}{m^{m}} .
$$

Then all solutions of the equation (1.1) oscillate.

\section{Remarks and examples}

Remark 4.1. It should be pointed out that the conditions (3.2) and (3.13) of Theorems 3.1 and 3.2 present for the first time sufficient conditions (in terms 
of lim sup) for the oscillation of all solutions to the equation (1.1) with several non-monotone arguments. They are also independent and essentially improve all the related oscillation conditions in the literature. Even in the case where $m=1$, the improvement is substantial.

Remark 4.2. Observe that when $m=1$, the above conditions (3.16) and (3.17) reduces to the (classical) condition (2.1).

The following examples illustrate the significance of our results.

Example 4.1. Let $a_{1}, \delta \in(0,+\infty)$ and consider the sequences $\left\{a_{k}\right\}_{n=1}^{+\infty}$, where $a_{k+1}=3+2 \delta+a_{k} \quad(k=1,2, \ldots)$. Choose $\alpha \in(0,1)$ and $\varepsilon>0$ such that

$$
\frac{\ln (1+e)}{e-\varepsilon}<\alpha<\ln 2
$$

Consider equation (1.2), where $\tau(t)=t-1$ and

$$
p(t)=\left\{\begin{array}{l}
\frac{1}{e} \quad \text { for } a_{k} \leq t \leq a_{k}+1 \\
\frac{\alpha e-1}{\delta e} t+\frac{\delta+\left(1+a_{k}\right)(1-\alpha e)}{\delta e} \\
\quad \text { for } a_{k}+1 \leq t \leq a_{k}+\delta+1 \\
\quad \text { for } a_{k}+\delta+1 \leq t \leq a_{k+1}-\delta \\
\frac{1-\alpha e}{\delta e} t+\frac{\alpha \delta e+\left(a_{k}+\delta+3\right)(\alpha e-1)}{\delta e} \\
\quad \text { for } a_{k+1}-\delta \leq t \leq a_{k+1} \\
\quad(k=1,2, \ldots) .
\end{array}\right.
$$

It is obvious that

$$
\liminf _{t \rightarrow+\infty} \int_{\tau(t)}^{t} p(s) d s=\frac{1}{e} \quad \text { and } \quad \lambda^{*}=e
$$

(see Lemma 2.1). By (4.2) and (4.1)

$$
\begin{aligned}
& \limsup _{t \rightarrow+\infty} \int_{\tau(t)}^{t} p(s) \exp \left\{\int_{\tau(s)}^{\tau(t)} p(s) d s\right\} \\
& \leq \limsup _{t \rightarrow+\infty} \int_{t-1}^{t} \alpha e^{\alpha(t-s)} d s=e^{\alpha}-1<1 .
\end{aligned}
$$


On the other hand by (4.1)-(4.3) we have

$$
\begin{gathered}
\limsup _{t \rightarrow+\infty} \int_{\tau(t)}^{t} p(s) \exp \left\{(e-\varepsilon) \int_{\tau(s)}^{\tau(t)} p(\xi) d \xi\right\} d s \\
=\limsup _{t \rightarrow+\infty} \int_{t-1}^{t} \alpha \exp \left\{(e-\varepsilon)^{\alpha(t-s)}\right\} d s \\
=\frac{1}{e-\varepsilon}\left(e^{\alpha(e-\varepsilon)}-1\right)>\frac{e}{e-\varepsilon}>1 .
\end{gathered}
$$

Therefore, according to (4.4) the condition (2.5) is not fulfilled. On the other hand by (4.5) the condition (3.13) holds a.e. and therefore all solutions of equation (1.2) oscillate.

Example 4.2 (cf. [3]). We consider a generalisation of an example presented in [3], where the equation

$$
x^{\prime}(t)+\frac{1}{e} x(\tau(t))=0, \quad t \geq 0,
$$

with the retarded argument

$$
\tau(t):= \begin{cases}t-1, & t \in[3 n, 3 n+1], \\ -3 t+(12 n+3), & t \in[3 n+1,3 n+2], \\ 5 t-(12 n+13), & t \in[3 n+2,3 n+3],\end{cases}
$$

was studied. Here we discuss the more general equation

$$
x^{\prime}(t)+p x(\tau(t))=0, \quad t \geq 0, p>0,
$$

and illustrate how our methodology can be utilized to prove the existence of oscillatory solutions for some range of the parameter $p$. In this case, as in [3], one may choose the function

$$
\sigma(t)= \begin{cases}t-1, & t \in[3 n, 3 n+1], \\ 3 n, & t \in[3 n+1,3 n+2.6], \\ 5 t-(12 n+13), & t \in[3 n+2.6,3 n+3] .\end{cases}
$$

Now note that, since $\tau(t) \leq t-1$,

$$
\int_{\tau(t)}^{t} p d u \geq \int_{t-1}^{t} p d u=p .
$$

The choice as in [3] of $t_{n}=3 n+3$ gives 


$$
\begin{aligned}
C & =\limsup _{t \rightarrow+\infty} \int_{\sigma(t)}^{t} p \exp \left(\int_{\tau(s)}^{\sigma(t)} p \exp \left(\int_{\tau(\xi)}^{\xi} p d u\right) d \xi\right) d s \\
& \geq \lim _{n \rightarrow+\infty} \int_{3 n+2}^{3 n+3} p \exp \left(\int_{5 s-(12 n+13)}^{3 n+2} p \exp (p) d \xi\right) d s=\frac{1}{5}\left(e^{5 p e^{p}}-1\right) e^{-p}
\end{aligned}
$$

The inequality

$$
\frac{1}{5}\left(e^{5 p e^{p}}-1\right) e^{-p}>1
$$

is satisfied for (the numbers that follow are rounded to the third decimal place unless exact) $p \in[0.303,0.358]$. Thus, for $p \in[0.303,0.358]$ the condition (3.14) of Corollary 2.1 is satisfied and therefore all solutions to the above equation (4.6) oscillate. Observe, however, that when $p \in[0.303,0.358]$ in (4.6), we find

$$
\begin{gathered}
A=\limsup _{t \rightarrow \infty} \int_{\sigma(t)}^{t} p d s=p \cdot(2.6)<1, \\
\mathfrak{a}:=\liminf _{t \rightarrow \infty} \int_{\tau(t)}^{t} p d s=p<\frac{1}{e}
\end{gathered}
$$

and

$$
\begin{aligned}
\int_{\sigma(3 n+3)}^{3 n+3} p \exp \left\{\int_{\tau(s)}^{\sigma(3 n+3)} p d \xi\right\} d s & =\int_{3 n+2}^{3 n+3} p \exp \left\{\int_{5 s-(12 n+13)}^{3 n+2} p d \xi\right\} d s \\
& =\frac{1}{5}\left(e^{5 p}-1\right)<1 .
\end{aligned}
$$

That is, none of the known oscillation conditions (2.1), (2.2) (and also the conditions (2.6)-(2.11)) and (2.5) is satisfied.

Remark 4.3. It is obvious that if for some $i_{0} \in\{1, \ldots, m\}$ all solutions of the equation

$$
x^{\prime}(t)+p_{i_{0}}(t) x\left(\tau_{i_{0}}(t)\right)=0
$$

oscillate, then all solutions of the equation (1.1) also oscillate.

Example 4.3. Let $p, \Delta_{1}, \Delta_{2} \in(0,+\infty)$ and consider the sequences $\left\{t_{k}\right\}_{k=1}^{\infty}$ such that $t_{k} \uparrow+\infty$ for $k \uparrow+\infty, t_{k}+2 \Delta<t_{k+1} \quad(k=1,2, \ldots)$, where $\Delta=$ $\max \left\{\Delta_{i}, i=1,2\right\}$. Choose $p, \Delta_{1}$ and $\Delta_{2}$ such that

$$
p^{2} \Delta_{1} \Delta_{2}>\frac{1}{4}
$$


and

$$
p \Delta_{i}<1 \quad(i=1,2) .
$$

Let $p(t)=p$ for $t \in\left[t_{k}, t_{k}+\Delta\right](k=1,2, \ldots)$ and $p(t)=0$ for $t \in R_{+} \backslash U_{k=1}^{\infty}\left[t_{k}\right.$, $\left.t_{k}+\Delta\right]$.

According to (4.7) it is obvious that the condition (3.18) is fulfilled, where $m=2$ and $\tau_{i}(t)=t-\Delta_{i}(i=1,2)$, a.e. and therefore all solutions to equation (1.1) are oscillatory. However, for the equations

$$
x^{\prime}(t)+p(t) x\left(t-\Delta_{i}\right)=0 \quad(i=1,2)
$$

by (4.8), we have

$$
\limsup _{t \rightarrow+\infty} \int_{t-\Delta_{i}}^{t} p(s) d s<1 \quad(i=1,2)
$$

and

$$
\liminf _{t \rightarrow+\infty} \int_{t-\Delta_{i}}^{t} p(s) d s=0 \quad(i=1,2) .
$$

Remark 4.4. In the above mentioned Example 4.3, by a solution, we mean an absolutely continuous function which satisfies the corresponding equation almost everywhere.

Example 4.4. Consider the equation

$$
x^{\prime}(t)+p_{1} x\left(\tau_{1}(t)\right)+p_{2} x\left(\tau_{2}(t)\right)=0, \quad t \geq 0, p_{1}, p_{2}>0,
$$

where

$$
\begin{gathered}
\tau_{1}(t)= \begin{cases}t-1, & t \in[3 n, 3 n+1], \\
-3 t+(12 n+3), & t \in[3 n+1,3 n+2], \\
5 t-(12 n+13), & t \in[3 n+2,3 n+3],\end{cases} \\
\tau_{2}(t)= \begin{cases}t-2, & t \in[3 n, 3 n+1], \\
-t+6 n, & t \in[3 n+1,3 n+2], \\
3 t-(6 n+8), & t \in[3 n+2,3 n+3] .\end{cases}
\end{gathered}
$$

We can take

$$
\begin{gathered}
\sigma_{1}(t)= \begin{cases}t-1, & t \in[3 n, 3 n+1], \\
3 n, & t \in[3 n+1,3 n+2.6], \\
5 t-(12 n+13), & t \in[3 n+2.6,3 n+3],\end{cases} \\
\sigma_{2}(t)= \begin{cases}t-2, & t \in[3 n, 3 n+1], \\
3 n-1, & t \in[3 n+1,3 n+2 . \overline{3}], \\
3 t-(6 n+8), & t \in[3 n+2 . \overline{3}, 3 n+3] .\end{cases}
\end{gathered}
$$


Note that, since $\tau_{1}(t) \leq t-1$ and $\tau_{2}(t) \leq t-2$, we have

$$
\int_{\tau_{1}(t)}^{t} d u \geq \int_{t-1}^{t} d u=1, \quad \int_{\tau_{2}(t)}^{t} d u \geq \int_{t-2}^{t} d u=2 .
$$

Set $P=p_{1} \exp \left(p_{1}+p_{2}\right)+p_{2} \exp \left(2 p_{1}+2 p_{2}\right)$. The choice of $t_{n}=3 n+3$ gives

$$
\begin{aligned}
& \limsup _{t \rightarrow+\infty} \prod_{j=1}^{2}\left(\prod_{i=1}^{2} \int_{\sigma_{j}(t)}^{t} p_{i} \exp \left(\int_{\tau_{i}(s)}^{\sigma_{i}(t)} \sum_{i=1}^{2} p_{i} \exp \left(\int_{\tau_{i}(\xi)}^{\xi}\left(p_{1}+p_{2}\right) d u\right) d \xi\right) d s\right)^{1 / 2} \\
& \geq \lim _{n \rightarrow+\infty} \prod_{j=1}^{2}\left(\prod _ { i = 1 } ^ { 2 } \int _ { \sigma _ { j } ( 3 n + 3 ) } ^ { 3 n + 3 } p _ { i } \operatorname { e x p } \left(\int_{\tau_{i}(s)}^{\sigma_{i}(3 n+3)} \sum_{i=1}^{2} p_{i}\right.\right. \\
& \left.\left.\times \exp \left(\int_{\tau_{i}(\xi)}^{\xi}\left(p_{1}+p_{2}\right) d u\right) d \xi\right) d s\right)^{1 / 2} \\
& \geq \lim _{n \rightarrow+\infty} \prod_{j=1}^{2}\left(\int_{\sigma_{j}(3 n+3)}^{3 n+3} p_{1} \exp \left(\int_{\tau_{1}(s)}^{3 n+2} P d \xi\right) d s\right)^{1 / 2} \\
& \times\left(\int_{\sigma_{j}(3 n+3)}^{3 n+3} p_{2} \exp \left(\int_{\tau_{2}(s)}^{3 n+1} P d \xi\right) d s\right)^{1 / 2} \\
& =\lim _{n \rightarrow+\infty}\left(\int_{3 n+2}^{3 n+3} p_{1} \exp \left(\int_{\tau_{1}(s)}^{3 n+2} P d \xi\right) d s\right)^{1 / 2} \times\left(\int_{3 n+2}^{3 n+3} p_{2} \exp \left(\int_{\tau_{2}(s)}^{3 n+1} P d \xi\right) d s\right)^{1 / 2} \\
& \times\left(\int_{3 n+1}^{3 n+3} p_{1} \exp \left(\int_{\tau_{1}(s)}^{3 n+2} P d \xi\right) d s\right)^{1 / 2} \times\left(\int_{3 n+1}^{3 n+3} p_{2} \exp \left(\int_{\tau_{2}(s)}^{3 n+1} P d \xi\right) d s\right)^{1 / 2} \\
& =\lim _{n \rightarrow+\infty}\left(\int_{3 n+2}^{3 n+3} p_{1} \exp \left(\int_{5 s-(12 n+13)}^{3 n+2} P d \xi\right) d s\right)^{1 / 2} \\
& \times\left(\int_{3 n+2}^{3 n+3} p_{2} \exp \left(\int_{3 s-(6 n+8)}^{3 n+1} P d \xi\right) d s\right)^{1 / 2} \\
& \times\left(\int_{3 n+1}^{3 n+2} p_{1} \exp \left(\int_{-3 s+(12 n+3)}^{3 n+2} P d \xi\right) d s\right. \\
& \left.+\int_{3 n+2}^{3 n+3} p_{1} \exp \left(\int_{5 s-(12 n+13)}^{3 n+2} P d \xi\right) d s\right)^{1 / 2}
\end{aligned}
$$




$$
\begin{aligned}
& \times\left(\int_{3 n+1}^{3 n+2} p_{2} \exp \left(\int_{-s+6 n}^{3 n+1} P d \xi\right) d s+\int_{3 n+2}^{3 n+3} p_{2} \exp \left(\int_{3 s-(6 n+8)}^{3 n+1} P d \xi\right) d s\right)^{1 / 2} \\
= & : D\left(p_{1}, p_{2}\right) .
\end{aligned}
$$

Let $p_{1}=0.1$ then, by direct computation, we get

$$
D>\frac{1}{4}
$$

if $p_{2} \geq 0.158$. That is, when $p_{1}=0.1$ and $p_{2} \geq 0.158$ in equation (4.9), the condition (3.1) of Theorem 3.1 is satisfied and therefore all solutions to this equation oscillate.

Note that since the delays are not monotone, Theorem 2.5 cannot be applied to this example. We now compare our result with Theorem 2.4. Note that

$$
\tau_{1}(t), \tau_{2}(t) \leq \sigma_{1}(t) \quad \text { for every } t>0 .
$$

The choice $p_{1}=0.1, p_{2}=0.158$ gives

$$
\liminf _{t \rightarrow \infty} \int_{\sigma_{1}(t)}^{t}\left(p_{1}+p_{2}\right) d s=p_{1}+p_{2}=0.258<\frac{1}{e} .
$$

\section{Acknowledgement}

This paper was partially written during a visit of $G$. Infante to the Department of Mathematics of the University of Ioannina. G. Infante is grateful to the people of the aforementioned Department for their kind and warm hospitality. The work was supported by the Sh. Rustaveli National Science Foundation. Grant No. 31/09.

\section{References}

[ 1 ] Arino, O., Gyori, I. and Jawhari, A., Oscillation criteria in delay equations, J. Differential Equations, 53 (1984), 115-123.

[2] Berezansky, L. and Braverman, E., On some constants for oscillation and stability of delay equations, Proc. Amer. Math. Soc., 139 (2011), 4017-4026.

[3] Braverman, E. and Karpuz, B., On oscillation of differential and difference equations with non-monotone delays, Appl. Math. Comput., 218 (2011), 3880-3887.

[4] Elbert, A. and Stavroulakis, I. P., Oscillations of first order differential equations with deviating arguments, Recent trends in differential equations, World Sci. Ser. Appl. Anal., 1, World Sci. Publ., River Edge, NJ, 1992, pp. 163-178.

[ 5] Elbert, A. and Stavroulakis, I. P., Oscillation and nonoscillation criteria for delay differential equations, Proc. Amer. Math. Soc., 123 (1995), 1503-1510. 
[6] Erbe, L. H., Kong, Qingkai and Zhang, B. G., Oscillation Theory for Functional Differential Equations, Monographs and Textbooks in Pure and Applied Mathematics, 190, Marcel Dekker, Inc., New York, 1995.

[ 7 ] Erbe, L. H. and Zhang, B. G., Oscillation for first order linear differential equations with deviating arguments, Differential Integral Equations, 1 (1988), 305-314.

[ 8 ] Fukagai, N. and Kusano, T., Oscillation theory of first order functional differential equations with deviating arguments, Ann. Mat. Pura Appl. (4), 136 (1984), 95-117.

[9] Gopalsamy, K., Stability and Oscillations in Delay Differential Equations of Population Dynamics, Mathematics and its Applications, 74, Kluwer Academic Publishers Group Dordrecht, 1992.

[10] Grammatikopoulos, M. K., Koplatadze, R. G. and Stavroulakis, I. P., On the oscillation of solutions of first order differential equations with retarded arguments, Georgian Math. J., 10 (2003), 63-76.

[11] Gyori, I. and Ladas, G., Oscillation Theory of Delay Differential Equations with Applications, Oxford Mathematical Monographs, Oxford Science Publications, The Clarendon Press, Oxford University Press, New York, 1991.

[12] Hale, J. K., Theory of Functional Differential Equations, Second edition, Applied Mathematical Sciences, Vol. 3, Springer-Verlag, New York-Heidelberg, 1977.

[13] Hunt, B. R. and Yorke, J. A., When all solutions of $x^{\prime}=\sum q_{i}(t) x\left(t-T_{i}(t)\right)$ oscillate, J. Differential Equations, 53 (1984), 139-145.

[14] Jaroš, J. and Stavroulakis, I. P., Oscillation tests for delay equations, Rocky Mountain J. Math., 29 (1999), 197-207.

[15] Jian, C., Oscillation of linear differential equations with deviating argument, Math. Practice Theory, 1 (1991), 32-41 (in Chinese).

[16] Kon, M., Sficas, Y. G. and Stavroulakis, I. P., Oscillation criteria for delay equations, Proc. Amer. Math. Soc., 128 (2000), 2989-2997.

[17] Koplatadze, R. G. and Chanturija, T. A., On the oscillatory and monotonic solutions of first order differential equations with deviating arguments, Differentsial'nye Uravneniya, 18 (1982), 1463-1465.

[18] Koplatadze, R. G. and Kvinikadze, G., On the oscillation of solutions of first order delay differential inequalities and equations, Georgian Math. J., 1 (1994), 675-685.

[19] Kwong, M. K., Oscillation of first-order delay equations, J. Math. Anal. Appl., 156 (1991), 274-286.

[20] Ladas, G., Sharp conditions for oscillations caused by delay, Applicable Anal., 9 (1979), 93-98.

[21] Ladas, G., Laskhmikantham, V. and Papadakis, J. S., Oscillations of higher-order retarded differential equations generated by the retarded arguments, Delay and Functional Differential Equations and Their Applications, Academic Press, New York, 1972, pp. 219-231.

[22] Ladas, G. and Stavroulakis, I. P., Oscillations caused by several retarded and advanced arguments, J. Differential Equations, 44 (1982), 134-152.

[23] Ladde, G. S., Lakshmikantham, V. and Zhang, B. G., Oscillation Theory of Differential Equations with Deviating Arguments, Monographs and Textbooks in Pure and Applied Mathematics, 110, Marcel Dekker, Inc., New York, 1987.

[24] Li, B., Oscillations of first order delay differential equations, Proc. Amer. Math. Soc., 124 (1996), 3729-3737.

[25] Myshkis, A. D., Linear homogeneous differential equations of first order with deviating arguments, Uspehi Matem. Nauk (N.S.), 5 (1950), 160-162 (Russian). 
[26] Sficas, Y. G. and Stavroulakis, I. P., Oscillation criteria for first-order delay equations, Bull. London Math. Soc., 35 (2003), 239-246.

[27] Wang, Z. C., Stavroulakis, I. P. and Qian, X. Z., A survey on the oscillation of solutions of first order linear differential equations with deviating arguments, Appl. Math. E-Notes, 2 (2002), 171-191.

[28] Yu, J. S., Wang, Z. C., Zhang, B. G. and Qian, X. Z., Oscillations of differential equations with deviating arguments, Panamer. Math. J., 2 (1992), 59-78.

[29] Zhou, Y. and Yu, Y. H., On the oscillation of solutions of first order differential equations with deviating arguments, Acta Math. Appl. Sinica (English Ser.), 15 (1999), 297-302.

\author{
nuna adreso: \\ G. Infante \\ Dipartimento di Matematica ed Informatica \\ Università della Calabria \\ 87036 Arcavacata di Rende, Cosenza \\ Italy \\ E-mail: gennaro.infante@unical.it \\ R. Koplatadze \\ Department of Mathematics \\ Tbilisi State University \\ Tbilisi \\ Georgia \\ E-mail: r_koplatadze@yahoo.com \\ I. P. Stavroulakis \\ Department of Mathematics \\ University of Ioannina \\ 45110 Ioannina \\ Greece \\ E-mail: ipstav@cc.uoi.gr
}

(Ricevita la 25-an de septembro, 2013)

(Reviziita la 10-an de decembro, 2013) 